\title{
Type-Based Query Expansion for Sentence Retrieval
}

\author{
Keke Cai, Chun Chen, Jiajun Bu, and Guang Qiu \\ College of Computer Science, Zhejiang University \\ Hangzhou, 310027, China \\ \{caikeke, chenc, bjj, qiuguang\}@zju.edu.cn
}

\begin{abstract}
In this paper, a novel sentence retrieval model with type-based expansion is proposed. In this retrieval model, sentences expected to be relevant should meet with the requirements both in query terms and query types. To obtain the information about query types, this paper proposes a solution based on classification, which utilizes the potential associations between terms and information types to obtain the optimized classification results. Inspired by the idea that relevant sentences always tend to occur nearby, this paper further reranks each sentence by considering the relevance of its adjacent sentences. The proposed retrieval model has been compared with other traditional retrieval models and experiment results indicate its significant improvements in retrieval effectiveness.
\end{abstract}

Keywords: Sentence retrieval, query type identification, query expansion.

\section{Introduction}

Sentence retrieval is to retrieve query-relevant sentences in response to users' queries. It has been widely applied in many traditional applications, such as passage retrieval [1], document summarization [2], question answering [3], novelty detection [4] and content-based retrieval presentation [5]. A lot of different approaches have been proposed for sentence retrieval. Most of them, however, have not been proven efficient enough. The main reason is due to the limited information expressed in sentences. To improve the performance of sentence retrieval, besides the key words in queries and sentences, additional features that are helpful for indicating sentences' relevance should be explored.

Query type, which expresses relevant information satisfying users' information need, has been effectively used in some applications involving sentence retrieval, such as question-answering that looks for sentences containing the expected type of answer, and novelty detection where sentences involving information of special type will be considered more relevant. However, little efforts have been made to incorporate such information into the process of keyword-based information retrieval, where the most difficulty is the identification of query-relevant information types.

This paper proposes a new sentence retrieval model, in which the information about query types is explored and incorporated into the retrieval process. The idea is similar to that of query expansion. The difference is that this model expands each query with the relevant information types instead of the concreted terms. In this paper, 
query types are defined as the types of the expected information entities, such as persons, locations, numbers, dates, times and etc, which are considered necessary for satisfying user's request or information need. Therefore, in the retrieval process, sentences expected to be relevant should meet with the requirements both in query terms and query types. To achieve such a retrieval model, the most important factor is the identification of query types. This paper proposes a solution based on classification. This classification model makes a full use of the theory of information association, with the purpose to utilize the potential associations between terms and information types to obtain the optimized classification results. In addition to term and type information described above, another type of information is also considered in the evaluation of sentence relevance, that is, the proximity between sentences. The idea underneath is that relevant sentences always tend to occur nearby. Then, each sentence is further re-ranked by considering the relevance of its adjacent sentences.

The remainder of the paper is structured as follows: Section 2 introduces the related studies in sentence retrieval. Section 3 describes the proposed sentence retrieval model and the classification approach for query type identification. In Section 4, the experimental results are presented. Section 5 concludes the paper.

\section{Related Works}

Most exiting approaches for sentence retrieval are based on term matching between query and sentence. They are essentially the applications of algorithms designed for document retrieval [6] [7] [8]. However, compared with documents, sentences are much smaller. Thus, the performance of typical document retrieval systems on the retrieval of sentences is significantly worse. Some systems try to utilize linguistic or other features of sentences to facilitate the detection of relevant sentences. In the study of [5], factors used for ranking sentences include the position of sentence in the source document, the words contained in sentence and the number of query terms contained in sentence. In [9], semantic and lexical features are extracted from the initial retrieved sentences to filter out possible non-relevant sentences.

In addition to the mining of features in sentences, some systems concentrate on the studies of features in queries. One of the most significant features about queries is the query type. In most cases, query type is defined as the entity types of the expected answer. For example, in [4], queries are described as patterns that include both query words and the required answer types. Then, these patterns are used to retrieve sentences. Sentences without the expected type of named entities will be considered irrelevant. In the domain of question answering, query type is also an important factor for sentence relevance evaluation. Given a question, the question is analyzed for their expected answer type and then submitted to retrieve sentences that contain the key words from the question or the tokens or phrase that are consistent with the expected answer type of the question. Studies have shown the positive effects of query type on sentence retrieval, which however, in the context of keyword-based retrieval, has not been fully utilized. The most difficulty is the identification of query types, which becomes one of the focuses of our studies in this paper. 


\section{Sentence Retrieval Model Involved with Query Type}

The proposed sentence retrieval model with type-based query expansion measures sentence relevance from two perspectives: lexical similarity and type similarity. Lexical similarity is an indicator of the degree to which query and sentence discuss the same topic. It is always evaluated according to term matching between query and sentence. Given two terms, their similarity can be viewed from different point of views, such as, synonymy, variant, co-occurrence or others. Since this paper expects to pay more attention to the application of query type, we adopt the most basic definition for lexical similarity. If two terms are exactly the same, they are lexical similarity. Type similarity is actually to evaluate the coincidence between the query types and information types related to sentence. From sentence perspective, the related information types are defined as the types of entities contained in sentence and can be identified by using the existing named entity recognition technique. However, from query perspective, the identification of query types is a little more difficult. In this paper, this problem is solved by a solution based on classification.

\subsection{Information Association Based Classification}

Inspired by the theory of Hyperspace Analogue to Language (HAL) [10], a novel classification approach is proposed to solve the problem of query type identification. In this approach, a special type of information association is explored, with the purpose of reflecting the dependencies between terms and information types. When such kind of associations is incorporated into query classification, information types that have the most probabilities to be associated with a query can be identified. The implementation of this classification is based on the information model reflecting the Associations between Terms and Information Types (ATIT).

Construction of the ATIT Model. The construction of the ATIT model consists of two steps.

The first step is to construct the HAL model given the large document corpus. The HAL model is constructed in the traditional way (See [10] for more details). Let $T=$ $\left\{t_{1}, t_{2} \ldots t_{n}\right\}$ be the term set of the document corpus, the constructed HAL is finally represented as a $n^{*} n$ matrix $M_{H A L}$, in which each term of $T$ is described as a $n$ dimension term vector $V_{i}^{H A L}=\left\{M_{H A L}(i, 1), \ldots, M_{H A L}(i, n)\right\}$, where $M_{H A L}(i, j)$ describes the association strength between the terms $t_{i}$ and $t_{j}$.

The second step realizes the construction of ATIT model based on HAL model. In the ATIT model, each term $t_{i}$ is expected to be described as a $m$-dimension vector $V_{i}^{A T I T}=\left\{M_{\text {ATIT }}(i, 1), \ldots, M_{\text {ATIT }}(i, m)\right\}$, where $M_{\text {ATIT }}(i, j)$ represents the association strength between the term $t_{i}$ and the information type $c_{j}$. The construction of ATIT can be further divided into three sub-steps: Firstly, entity type related to each term $t_{i}$ is discovered. In this paper, it is realized by named entity recognition (NER) [11]. However, it is noted that not all entities of all types can be well identified. To solve this problem, the manual NER approach is adopted, which is to use human generated entity annotation results to realize a part of these entity recognitions. Secondly, based on the association information provided by HAL, the association strengths between 
terms and information types are calculated. Let terms of information type $c_{j}$ be $T_{c_{j}}=$ $\left\{t_{c_{j} l}, \ldots, t_{c_{j} k}\right\}$, the association strength between term $t_{i}$ and $c_{j}$ is calculated as:

$$
M_{A T I T}(i, j)=\sum_{p=c_{j} l}^{c_{j} k} M_{H A L}(i, p)
$$

Implementation of Classification. The ATIT information model makes it possible to identify the relationships between query terms and information types. Then, the probability of query $Q$ being relevant to information type $c_{j}$ can be evaluated by:

$$
P\left(c_{j} \mid Q\right)=\sum_{t_{i} \in Q} P\left(c_{j} \mid t_{i}\right) P\left(t_{i} \mid Q\right) .
$$

where $P\left(t_{i} \mid Q\right)$ means the probabilities of $t_{i}$ in $Q$. Since that queries are normally short, each $P\left(t_{i} \mid Q\right)$ can be approximately assigned an equal value, i.e., $P\left(t_{i} \mid Q\right)=1 /|Q|$, where $|Q|$ is the number of terms in the query. The probability $P\left(c_{j} \mid t_{i}\right)$ represents the association strength of the information type $c_{j}$ with respect to the term $t_{i}$. According to Bayesian formula, it can be transformed into:

$$
P\left(c_{j} \mid t_{i}\right)=\frac{P\left(t_{i} \mid c_{j}\right) * P\left(c_{j}\right)}{P\left(t_{i}\right)} .
$$

where $P\left(c_{j}\right)$ and $P\left(q_{i}\right)$ are respectively the prior probability of category $c_{j}$ and query term $q_{i}$. Here, we set them to be constants. $P\left(t_{i} \mid c_{j}\right)$ represents the conditional probability of $t_{i}$. Based on the previous constructed ATIT model, it is defined as:

$$
P\left(q_{i} \mid c_{j}\right)=\frac{M_{A T I T}(i, j)}{\sum_{i=1}^{n} M_{A T I T}(i, j)} .
$$

Probability of query $Q$ being relevant to information type $c_{j}$ can be evaluated by:

$$
P\left(c_{j} \mid Q\right) \stackrel{\text { rank }}{=} \sum_{t_{i} \in Q} \frac{M_{A T I T}(i, j)}{\sum_{i=1}^{n} M_{A T I T}(i, j)} .
$$

\subsection{Relevance Ranking of Sentence}

Experiments in [12] show that although there is no significant difference in the performance of traditional retrieval models when implemented in sentence retrieval, TFIDF technique performs consistently better than the others across different query 
sets. Thus, in this paper, we decided to use the vector space model (VSM) with tf.idf weighting as the retrieval framework. The retrieval model is formulated as:

$$
\operatorname{sim}(S, Q)=\lambda \sum_{l \in L_{S} \wedge L_{Q}} W_{S, l} * W_{Q, l}+(1-\lambda) \sum_{t \in T_{S} \wedge T_{Q}} W_{S, t} * W_{Q, t} .
$$

where, the parameter $\lambda$ is used to control the influence of each component to sentence retrieval. Let $A$ be the sentence $S$ or the query $Q$, then $L_{A}$ and $T_{A}$ respectively represent the term vector and the type vector with respect to $A$. $W_{A, l}$ denotes the weight of term $l$ in $A$ and can be defined as $\log \left(L t f_{l}+1\right) * \log \left(N / L d f_{l}+1\right)$, where $L t f_{l}$ is the frequency of term $l$ in $A, N$ is the total number of sentences and $L d f_{l}$ is the number of sentences containing the term $l$. $W_{S, t}$ denotes the weight of the information type $t$ in sentence $S$ and can be defined as $\log \left(T t f_{t}+1\right) * \log \left(N / T d f_{t}+1\right)$, where $T t f_{t}$ is the frequency of entities in $S$ with type $t$ and $T d f_{t}$ is the number of sentences containing entities of type $t . W_{Q, t}$ denotes the weight of the information type $t$ in query $Q$. It is the normalized probability of each query type and defined as:

$$
W_{Q, t}=\frac{P(t \mid Q)}{\sum_{t \in T_{Q}} p(t \mid Q)} .
$$

In our relevant sentence retrieval model, a sentence that contains not only query terms but also the entities of the expected information types will be considered more relevant. This is the main difference between our type-based expansion retrieval model and other word-based approaches. Since this method makes an estimation of the most possible query context by query relevant information types, the more accurate relevance judgment is expected. However, it is noted that in most cases this approach is more effective for long sentences than short sentences. To solve this problem, further optimization is considered. Conclusions in [9] show that query relevant information always appear in several continuous sentences. Inspired by this idea, this paper proposes to re-rank the sentence according to the following rules.

For sentence $S_{i}$ that has information of the expected types, but contain few query terms, if the sentence $S_{j}$ that is before or after $S_{i}$ has many terms in common with the query, but do not contain the expected information types, the ranks of sentences $S_{i}$ and $S_{j}$ will be improved by: $\operatorname{sim}^{\prime}\left(S_{i}, Q\right)=\operatorname{sim}\left(S_{i}, Q\right)+\alpha^{*} \operatorname{sim}\left(S_{j}, Q\right), \operatorname{sim}^{\prime}\left(S_{j}, Q\right)=\operatorname{sim}\left(S_{j}\right.$, $Q)+\beta^{*} \operatorname{sim}\left(S_{i}, Q\right)$, where $0 \leq \alpha, \beta \leq 1$ and $\operatorname{sim}\left(S_{i}, Q\right)$ and $\operatorname{sim}\left(S_{j}, Q\right)$ are the initial relevance values of $S_{i}$ and $S_{j}$ evaluated by formula 6 .

\section{Experiments}

We use the collections of the TREC Novelty Track 2003 and 2004 for the evaluation of our proposed retrieval method. To validate its applicability to keyword-based retrieval, we select the TREC topic titles to formulate the queries.

The first part of experiments is to verify the potential associations between query and information of certain types. Table 1 gives the statistical results. In this table, the second and third row shows the distribution of entity information in relevant sentences. Signification information about information entities has been discovered in 
most relevant sentences. It implies its large potentialities in sentence retrieval. In Table $1, \mathrm{P}(\mathrm{N})$ represents the probability of queries concerned with $\mathrm{N}$ types of entities. It is discovered that most queries involve one or two different types of information entities. This further approves the assumption of the underlying associations between query and information types, which, when considered carefully, will improve the effectiveness of sentence retrieval.

Table 1. Statistical information about entities and entity types in relevant sentences

\begin{tabular}{|c|c|c|c|c|c|c|c|c|}
\hline & \multicolumn{4}{|c|}{ TREC 2003} & \multicolumn{4}{|c|}{ TREC 2004} \\
\hline $\begin{array}{l}\text { \# of relevant sentences } \\
\text { per query }\end{array}$ & \multicolumn{4}{|c|}{311.14} & \multicolumn{4}{|c|}{166.86} \\
\hline $\begin{array}{l}\text { \# of entities per relevant } \\
\text { sentence }\end{array}$ & \multicolumn{4}{|c|}{1.703} & \multicolumn{4}{|c|}{1.935} \\
\hline \multirow[t]{2}{*}{$\mathrm{P}(\mathrm{N})$} & $\mathrm{N}=0$ & $\mathrm{~N}=1$ & $\mathrm{~N}=2$ & $\mathrm{~N}>2$ & $\mathrm{~N}=0$ & $\mathrm{~N}=1$ & $\mathrm{~N}=2$ & $\mathrm{~N}>2$ \\
\hline & $18 \%$ & $38 \%$ & $30 \%$ & $14 \%$ & $32 \%$ & $30 \%$ & $26 \%$ & $12 \%$ \\
\hline
\end{tabular}

Another part of experiments is to compare the classification performance of our proposed information association based approach (IA) with word pattern based approach (WP) applied in [4] and the machine learning classification approach (ML), which in this paper is support vector machine approach. We select the data obtained at the website (http://12r.cs.uiuc.edu/ cogcomp/Data/QA/QC/) as the training and test data. Since these data are in the form of question, to make them applicable to our classification case, we have transformed them to be the form of keyword query. These formed queries contain the kernel terms in questions excepting the interrogative terms. Table 2 respectively shows the experimental results measured by macro precision, macro recall and macro F1-measure. As shown in Table 2, word pattern based approach has a higher classification precision, but a lower recall. It on one hand shows the relative accuracy of the defined word pattern and on the other hand its limited coverage. Compared with the machine learning, our proposed approach has a similar classification precision, but achieves a higher recall. In our experiments, we discovered that some valued relationships between term and information type have not been identified as expected. It is for that reason that a large amount of needed entities information cannot be properly recognized by the applied entity recognition technique. The study of entity recognition technique is out of the scope of this paper. However, it is believed that with the perfection of entity recognition technique, information association based classification approach can achieve better performance.

The purpose of the results shown in Tables 3 is to compare the performance of the proposed sentence retrieval model with type-based query expansion (TPQE) to other three retrieval approaches, including TFIDF model (TFIDF), BIR model (BIR), KLdivergence model (KLD) and the traditional term-based expansion sentence retrieval model (TQE). Statistical analysis of the retrieval results shows that in the context of sentence retrieval the application of traditional document retrieval algorithm is actually to detect the existence of query terms in sentence or not. As shown in Table 3 there is no significant difference in the performances of these traditional retrieval models. However, since most sentences have fewer words, TQE approach always add noise to the sentence retrieval process. Sentences containing expansion terms may not 
be as relevant to the original query as sentences containing the original query terms. Table 3 shows that performance of this approach. Comparatively speaking, our proposed method considers query expansion from another perspective. It is helpful to identify the most relevant information of query and therefore avoids the introduction of large noise. As shown in Table 3, our proposed approach does do better than all other approaches.

Table 2. Performances of different classification approaches

\begin{tabular}{|l|l|l|l|}
\hline & Macro precision & Macro recall & Macro F1 \\
\hline WP & 0.7230 & 0.4418 & 0.4990 \\
\hline ML & 0.6980 & 0.5181 & 0.5778 \\
\hline IA & 0.6895 & 0.5903 & 0.6081 \\
\hline
\end{tabular}

Table 3. Performance comparison in finding relevant sentences for 50 queries in TREC 2003 \& TREC 2004

\begin{tabular}{|l|l|l|}
\hline Database & 50 queries in TREC 2003 & 50 queries in TREC 2004 \\
\hline TFIDF & 0.349 & 0.228 \\
\hline BIR & 0.292 & 0.178 \\
\hline KLD & 0.330 & 0.2833 \\
\hline TQE & 0.304 & 0.212 \\
\hline TPQE & 0.385 & 0.249 \\
\hline
\end{tabular}

Table 4. Retrieval performance with or without consideration of proximity

\begin{tabular}{|c|c|c|c|c|}
\hline Database & \multicolumn{2}{|c|}{50 queries in TREC 2003} & \multicolumn{2}{|c|}{50 queries in TREC 2004} \\
\hline Methods & TPQE & TPQE_PX & TPQE & TPQE_PX \\
\hline P@5 & 0.6324 & 0.6989 & 0.3920 & 0.4380 \\
\hline $\mathrm{P} @ 10$ & 0.6559 & 0.6860 & 0.3875 & 0.4200 \\
\hline $\mathrm{P} @ 20$ & 0.5326 & 0.6639 & 0.3704 & 0.4020 \\
\hline $\mathrm{P} @ 30$ & 0.5919 & 0.6400 & 0.3680 & 0.4160 \\
\hline $\mathrm{P} @ 50$ & 0.5534 & 0.5896 & 0.3542 & 0.3976 \\
\hline P@100 & 0.5026 & 0.5438 & 0.3185 & 0.3413 \\
\hline
\end{tabular}

With the hypothesis that relevant sentences always exist in close proximity to each other, we further propose the proximity-based optimization method for re-ranking the sentences. This optimization scheme focuses on the distributions of query terms and query types involved in the proximity sentences, with the hope to reveal some relevant sentences, which when work together, can provide the integrated information satisfying user's information need. Table 4 illustrates the experimental results, where P@n means the precision at the top $n$ ranked documents. As shown in this table 4, retrieval with consideration of sentence proximity achieves clear improvement in retrieval effectiveness. It further validates the relevancies among adjacent sentences. 


\section{Conclusion}

Compared with the traditional sentence retrieval model, the features of this proposed retrieval model include: it views the information of query type as a factor for identifying sentences' relevance; it re-ranks each sentence by considering the relationships between adjacent sentences. The proposed retrieval model has been compared with other traditional retrieval models. Experiment results indicate that it produces significant improvements in retrieval effectiveness.

\section{References}

1. Salton G., Allan J., Buckley C.: Automatic structuring and retrieval of large text files. Communication of the ACM, Vol. 37(2). (1994) 97-108

2. Daumé III H., Marcu D.: Bayesian query-focused summarization. In Proceedings of the $21^{\text {st }}$ International Conference on Computational Linguistics and $44^{\text {th }}$ Annual Meeting of the Association for Computational Linguistics. Sydney, Australia (2006) 305-312

3. Li X.: Syntactic Features in Question Answering. In Proceedings of $26^{\text {th }}$ Annual International ACM SIGIR Conference on Research and Development in Information Retrieval. Toronto, Canada. (2003) 383-38

4. Li X., Croft W.: Novelty detection based on sentence level patterns. In Proceedings of 2005 ACM CIKM International Conference on Information and Knowledge Management. Bremen, Germany. (2005) 744-751

5. White R., Jose J., Ruthven I.: Using top-ranking sentences to facilitate effective information access. Journal of the American Society for Information Science and Technology. Vol. 56(10). (2005) 1113-1125

6. Larkey L., Allan J., Connell M., Bolivar A., Wade, C.: UMass at TREC 2002: Cross Language and Novelty Tracks. In Proceedings of $11^{\text {th }}$ Text REtrieval Conference. Gaithersburg, Maryland. (2002) 721-732

7. Schiffman B.: Experiments in Novelty Detection at Columbia University. In Proceedings of $11^{\text {th }}$ Text REtrieval Conference. Gaithersburg, Maryland. (2002) 188-196

8. Zhang M., Lin C., Liu Y., Zhao L., Ma S.: THUIR at TREC 2003: Novelty, Robust and Web. In Proceedings of $12^{\text {th }}$ Text REtrieval Conference. Gaithersburg, Maryland. (2003) 556-567

9. Collins-Thompson K., Ogilvie P., Zhang Y., Callan J.: Information filtering, Novelty Detection, and Named-Page Finding. In Proceedings of $11^{\text {th }}$ Text REtrieval Conference. Gaithersburg, Maryland. 107-118

10. Lund K., Burgess C. Producing High dimensional Semantic Spaces from Lexical Cooccurrence. Behavior Research Methods, Instruments, \& Computers, Vol. 28, (1996) $203-$ 208

11. Andrew B. A Maximum Entropy Approach to Named Entity Recognition. Ph.D. thesis, New York University, (1999)

12. Allan J., Wade C., Bolivar A.: Retrieval and Novelty Detection at the Sentence Level. In Proceedings of $26^{\text {th }}$ Annual International ACM SIGIR Conference on Research and Development in Information Retrieval. Toronto, Canada. (2003) 314-321 